ISSN:2167-8685

\title{
On The Importance of Understanding Biointeractions of Nanomaterials with the Immune System
}

Simberg $\mathrm{D}^{1^{*}}$, Tsigelny $\mathrm{I}^{2,3,4}$

${ }^{1}$ The Skaggs School of Pharmacy and Pharmaceutical Sciences, University of Colorado, Anschutz Medical Campus, 12850 E. Montview Blvd., Aurora, CO, USA.

${ }^{2}$ San Diego Supercomputer Center, UCSD, MS 0505, 9500 Gilman Dr., La Jolla, CA, USA.

${ }^{3}$ Moores Cancer Center, UCSD, MS 0819, 3855 Health Sciences Dr., La Jolla, CA, USA.

${ }^{4}$ Department of Neurosciences UCSD, MS 0624, 9500 Gilman Dr., La Jolla, CA, USA.

\section{*Corresponding Author:}

Dmitri Simberg,

The Skaggs School of Pharmacy and Pharmaceutical Sciences, University of Colorado, Anschutz Medical Campus, 12850 E. Montview Blvd., Aurora, CO, USA.

E-mail: dmitri.simberg@ucdenver.edu

Received: March 18, 2014

Published: April 07, 2014

Citation: Simberg D. (2014). On The Importance of Understanding Biointeractions of Nanomaterials with the Immune System, Int J Nano Stud Technol, 03(02), 01-02. doi: http://dx.doi.org/10.19070/2167$8685-140005$ e

Copyright:Simberg $\mathbf{D}^{\odot} 2014$ This is an open-access article distributed under the terms of the Creative Commons Attribution License, which permits unrestricted use, distribution and reproduction in any medium, provided the original author and source are credited.

In this brief editorial we would like to express our opinion on one of the most urgent problems in the field of nanomedicine. A recent review by Weissleder and colleagues [1] nicely summarized this problem in the following paragraph: "Reviewing recent literature reveals a disequilibrium: an exponentially growing number of papers describe the synthesis of new nanomaterials, but relatively few manuscripts comprehensively investigate the biological behavior and/or advantages over existing materials. We clearly need more of the latter. Indeed, an argument could be made that all new nanomaterials should be accompanied by more comprehensive biological profiling, including cytometry analysis of cell distribution and other biological assays".

The same opinion was voiced by other leading experts in the field of nanomedicine $[2,3]$. It is definitely important to engineer and manufacture newnanomaterials with novel characteristics, precise physicochemical properties and accurate drug loading. However, we are convinced that basic research aimed at understanding the biological behavior of nanocarriers, such as interactions with immune proteins and extra- and intracellular immune receptors is critically important and is desperately needed in order to move the filed of nanomedicine forward.

One of the best examples of the existing disequilibrium is the role of the complement system (CS) in nanoparticle toxicity and clearance. CS accounts for about $5 \%$ of globulins in serum and is responsible for recognition, elimination and destruction of pathogens (Fig. 1). Activation of the CS triggers the release of $\mathrm{C} 3 \mathrm{a}$ and $\mathrm{C} 5 \mathrm{a}$,which are the most potent known proinflammatory molecules and anaphylatoxins. Opsonization of pathogen surface with $\mathrm{C} 3 \mathrm{~b}$ and $\mathrm{C} 1 \mathrm{q}$ can cause recognition and clearance by macrophages[4]. Many nanoparticulate systems have been shown to trigger the CS activation, [5-15] and it is doubtless that the ones that have not been tested in the relevant assays also do so, simply due to the fact that they "foreign" to the body. The importance of the CS is exemplified by the fact that several generations of dextran iron oxide nanoparticles for magnetic resonance imaging (Feridex, Combidex, Resovist) have been withdrawn from the market because of widespread infusion-related side effects due to the CS activation. At the same time, despite the critical role of the $\mathrm{CS}$, the mechanisms of the CS activation by nanomedicines are still poorly understood and have not been fully investigated. Thus, as of the date of writing this editorial, there are approximately 87,000 "nanoparticle" hits in PubMed. If we do the search using the "complement" keyword, there are 143,000 hits in the PubMed. If we combine "nanoparticle AND complement" in the search, there are only 263 hits. So, out of all the papers that deal with nanoparticles, only $0.3 \%$ of these papers (or less) somehow relate to the complement aspect. The same disequilibrium is notable in the research funding: the search of the NIH funding portfolio (http://projectreporter.nih.gov) revealed that while there are 65 projects that contain "nanoparticle AND complement" keyword, none of them specifically deals with mechanisms of complement activation of nanoparticles and strategies to prevent the phenomenon through chemistry/rational design.

Also underrepresented in the existing research portfolio are the mechanisms of recognition of nanoparticles by macrophages, which are arguably the most important cells responsible for rapid clearance of nanomedicines, the most undesirable and frustrating phenomenon in the nanomedicine field. The current strategy to avoid nanoparticle immune clearance is using the set of old generic rules on decorating the surface of nanoparticles with polymers. This coating has been shown to create a brush border around nanoparticles and is postulated to provide non-specific impermeable barrier that sterically prevents access of plasma proteins and cell receptors and thereby promotes long circulation time of nanoparticles [16, 17]. The best example is long-chain polyethylene glycol (PEG), albeit other hydrophilic polymers such as Pluronic F68 [18-20], Poloxamer (block copolymer of polyethylene oxide and polypropylene oxide [21]) and polyvinylpyrrolidone [22] have been used. The surface grafting with PEG has been widely employed with many types of nanoparticles and is being used in commercial liposomal doxorubicin formulation Doxil ${ }^{\mathrm{TM}}$. As a rule, increasing polymer thickness and density decreases the macrophage recognition and prolongs the circulation time [1623]. However, polymer coated nanoparticles are still recognizable 
Figure 1: Simberg and Tsigelny

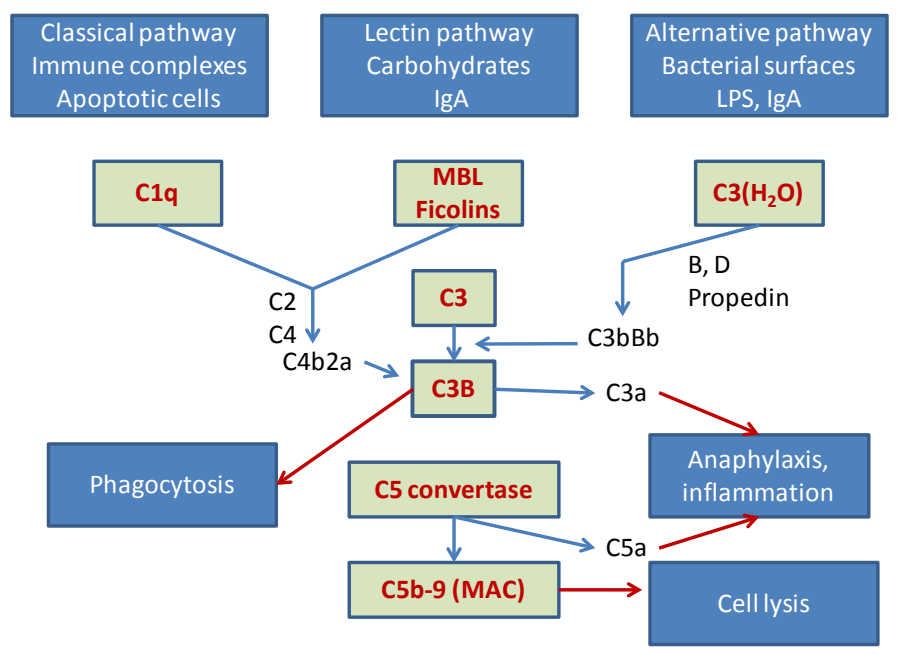

by liver and spleen macrophages (over $60-80 \%$ of the injected dose) and trigger complement activation. Another important shortcoming of the PEGylation is the apparent decrease in the affinity of surface tethered ligands due to the interference of the surrounding brush layer [24]. Small molecule (peptide)-mediated targeting should be especially sensitive due to ahigher chance of being buried and masked in the PEG brush layer. Another intrinsic limitation of PEGylation is the masking of nanoparticle's surface, mitigating potentially attractive properties of nanoparticles such as enzyme binding and activation, absorption of specific biomarkers, or change in optical properties.

We think it is high time for the nanomedicine field to take a step back and delve into the basic immunological aspects of interactions of all new and old nanomaterials with the biological milieu. This approach could be potentially rewarding as it could lead to important discoveries in order to advance the field that is still stuck in "PEG" paradigm[25]. The knowledge of how nanoparticles are recognized by immune proteins and receptors could be used for more efficient "camouflaging" of the nanoparticle surface, as compared to the empirical polymer coating. As a consequence, the increased circulation time can allow reducing the injected dose and achieving less deposition in the immune organs and more focal imaging/therapy compared with non-optimized nanoparticles. We predict that mechanistic studies of the bio-nano interface will enable nano-engineers and nano-chemists to perform rational (in silico?) design of nanoparticles with improved safety and efficacy.

\section{References}

[1]. Weissleder, R., Nahrendorf, M. \& Pittet, M. J. Imaging macrophages with nanoparticles. Nature materials 13, 125-138, (2014).

[2]. Moghimi, S. M., Hunter, A. C. \& Andresen, T. L. Factors controlling nanoparticle pharmacokinetics: an integrated analysis and perspective. Annual review of pharmacology and toxicology 52, 481-503, (2012).

[3]. Moghimi, S. M. \& Farhangrazi, Z. S. Nanomedicine and the complement paradigm. Nanomedicine : nanotechnology, biology, and medicine 9, 458460, (2013).

[4]. Taylor, P. R. et al. Macrophage receptors and immune recognition. Annu Rev Immunol 23, 901-944, (2005).

[5]. Szebeni, J. Complement activation-related pseudoallergy: a new class of drug-induced acute immune toxicity. Toxicology 216, 106-121, (2005).

[6]. Andersen, A. J., Hashemi, S. H., Andresen, T. L., Hunter, A. C. \& Moghimi, S. M. Complement: alive and kicking nanomedicines. J Biomed Nanotechnol 5, 364-372, (2009).

[7]. Pedersen, M. B. et al. Curvature of synthetic and natural surfaces is an im- portant target feature in classical pathway complement activation. Journal of immunology 184, 1931-1945, (2010).

[8]. Andersen, A. J. et al. Single-Walled Carbon Nanotube Surface Control of Complement Recognition and Activation. ACS Nano, (2013).

[9]. Peracchia, M. T., Vauthier, C., Passirani, C., Couvreur, P. \& Labarre, D. Complement consumption by poly(ethylene glycol) in different conformations chemically coupled to poly(isobutyl 2-cyanoacrylate) nanoparticles. Life Sci 61, 749-761, (1997).

[10]. Pham, C. T. et al. Variable antibody-dependent activation of complement by functionalized phospholipid nanoparticle surfaces. J Biol Chem 286, 123130, (2011).

[11]. Al-Hanbali, O., Rutt, K. J., Sarker, D. K., Hunter, A. C. \& Moghimi, S. M. Concentration dependent structural ordering of poloxamine 908 on polystyrene nanoparticles and their modulatory role on complement consumption. J Nanosci Nanotechnol 6, 3126-3133, (2006).

[12]. Moghimi, S. M., Hamad, I., Andresen, T. L., Jorgensen, K. \& Szebeni, J. Methylation of the phosphate oxygen moiety of phospholipidmethoxy(polyethylene glycol) conjugate prevents PEGylated liposome-mediated complement activation and anaphylatoxin production. Faseb J 20, 2591-2593, (2006).

[13]. Salvador-Morales, C., Zhang, L., Langer, R. \& Farokhzad, O. C. Immunocompatibility properties of lipid-polymer hybrid nanoparticles with heterogeneous surface functional groups. Biomaterials 30, 2231-2240, (2009).

[14]. Andersen, A. J. et al. Single-walled carbon nanotube surface control of complement recognition and activation. ACS Nano 7, 1108-1119, (2013).

[15]. Moore, A., Weissleder, R. \& Bogdanov, A., Jr. Uptake of dextran-coated monocrystalline iron oxides in tumor cells and macrophages. J Magn Reson Imaging 7, 1140-1145, (1997)

[16]. Alexis, F., Pridgen, E., Molnar, L. K. \& Farokhzad, O. C. Factors affecting the clearance and biodistribution of polymeric nanoparticles. Mol Pharm 5, 505-515, (2008).

[17]. Moghimi, S. M., Hunter, A. C. \& Murray, J. C. Long-circulating and target-specific nanoparticles: theory to practice. Pharmacol Rev 53, 283-318, (2001).

[18]. Chung, Y. I. et al. The effect of surface functionalization of PLGA nanoparticles by heparin- or chitosan-conjugated Pluronic on tumor targeting. J Control Release 143, 374-382, (2010).

[19]. Oh, K. S. et al. Paclitaxel-loaded Pluronic nanoparticles formed by a temperature-induced phase transition for cancer therapy. J Control Release, (2010).

[20]. Hsu, S. H. et al. Physicochemical characterization and in vivo bioluminescence imaging of nanostructured lipid carriers for targeting the brain: apomorphine as a model drug. Nanotechnology 21, 405101, (2010).

[21]. Esmaeili, F. et al. PLGA nanoparticles of different surface properties: preparation and evaluation of their body distribution. Int J Pharm 349, 249-255, (2008).

[22]. Gaur, U. et al. Biodistribution of fluoresceinated dextran using novel nanoparticles evading reticuloendothelial system. Int J Pharm 202, 1-10, (2000).

[23]. Perrault, S. D., Walkey, C., Jennings, T., Fischer, H. C. \& Chan, W. C. Mediating tumor targeting efficiency of nanoparticles through design. Nano Lett 9, 1909-1915, (2009).

[24]. Duncanson, W. J. et al. Targeted binding of PLA microparticles with lipidPEG-tethered ligands. Biomaterials 28, 4991-4999, (2007).

[25]. Verhoef, J. J. F. \& Anchordoquy, T. Questioning the use of PEGylation for drug delivery. Drug Deliv. and Transl. Res. 3, 499-503, (2013). 